\title{
Traum-Beruf oder Berufs-Trauma?
}

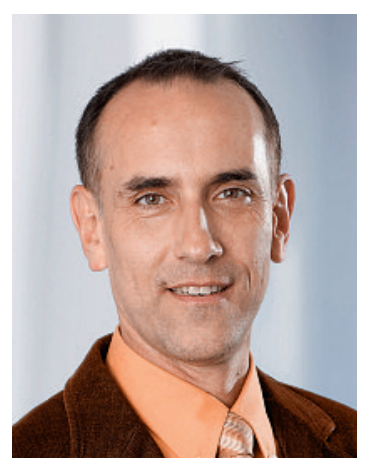

60 Arbeitsstunden pro Woche sind für Assistenz- und Oberärztinnen und -ärzte vielfach noch immer Alltag. Nicht selten sind sie zwölf Tage am Stück im Einsatz. Die langen und unregelmässigen Arbeitszeiten, Wochenendeinsätze, Nachtschichten, Pikettdienste, befristete Anstellungen u.a. machen es für Assistenz- und Oberärztinnen und -ärzte schwierig, Familie und Beruf unter einen Hut zu bringen. Oft verlängern unvorhergesehene und damit nicht planbare Ereignisse und Tätigkeiten den Arbeitstag. Dieser dauert nicht selten bis weit in den Abend oder in die Nacht hinein. Die meisten Kinderkrippen haben dann bereits geschlossen. Wohin also mit den Kindern? Für Spitalärztinnen und -ärzte mit Familie ein fast unlösbares Problem.

\section{Arztberuf und Familie lassen sich kaum kombinieren}

Eigentlich ist es erstaunlich, dass so wenig für die Vereinbarkeit von Arztberuf und Familie getan wird. Denn der Frauenanteil im stationären Sektor liegt heute bei über 40 Prozent, bei den Assistenzärzten sind bereits mehr als die Hälfte Frauen, und bei den Medizinstudenten sowie beim Staatsexamen liegt die Frauenquote bei über 60 Prozent. Viele Frauen steigen deshalb während der Weiterbildungszeit aus dem Arztberuf aus, spätestens jedoch dann, wenn sie Mutter werden. Denn wer hat noch genügend Energie, sich nach zwölf oder mehr Arbeitsstunden intensiv und ausreichend um das Wohl der Familie zu kümmern?

\section{Zu lange Arbeitszeiten schaden Ärzten und Patienten}

Solche Arbeitszeiten sind zudem ungesund, sie schaden nicht nur den Patienten, sondern vor allem auch den Ärztinnen und Ärzten selbst. Gemäss einer Studie aus Deutschland [1] dachte die Hälfte aller befragten Krankenhausärzte in den vergangenen zwölf Monaten darüber nach, den Beruf aufzugeben. Gleichzeitig kommt die Studie zum Schluss, dass rund 50 Prozent der Spitalärztinnen und -ärzte am Burn-outSyndrom leidet. Dabei sind Frauen, Berufsanfänger und Ärzte in niedrigen Positionen signifikant häufiger betroffen.
Neben einer massiv höheren Suizidrate - sie ist in der Ärzteschaft rund 40 Prozent höher als im Schnitt der Bevölkerung; bei den Ärztinnen liegt sie sogar 130 Prozent höher besteht auch eine grössere Suchtgefahr. Werden eigene körperliche Warnsignale ignoriert oder verdrängt, resultiert die «ideale Klientel» für ein Burn-out.

\section{Arbeitsgesetz muss endlich auch vollumfänglich für Assistenz- und Oberärzte umgesetzt werden}

Zwar ist seit 2005 eine Höchstarbeitszeit für Assistenzund Oberärzte von maximal 50 Stunden pro Woche gesetzlich vorgeschrieben und die Anzahl Arbeitstage am Stück wurde auf sieben begrenzt. Doch die Arbeitsrealität sieht oft anders aus. Die Liste widerrechtlicher Bedingungen stellt sich leider sehr umfangreich dar (z.B. Freitage werden nicht gewährt, Ferien und Pausen können nicht bezogen werden, Ruhezeiten beim Pikettdienst werden nicht berücksichtigt ...) und liesse sich fast beliebig fortsetzen. Die Betroffenen reichen nur selten Klage ein. Denn sie stehen insbesondere während der Weiterbildungszeit in einem Abhängigkeitsverhältnis. Lange Arbeitszeiten gelten zudem bei einigen Chefärzten immer noch als Ausdruck von Leistungsfähigkeit, Leistungswille und ärztlichem Ethos.

Wenn wir weiterhin Erfolge in Behandlung und Heilung von Krankheiten wünschen und die hohe Qualität des Schweizer Gesundheitssystems erhalten wollen, sind wir auf einfühlsame, gut ausgebildete und motivierte Ärzte angewiesen. Das Rüstzeug existiert bereits, es müsste lediglich praktiziert werden: hierzu gehört:

- die Einhaltung der Minimalstandards des Arbeitsgesetzes; - keine Überschreitung der wöchentlichen Höchstarbeitszeit von 50 Stunden, max. zulässige sieben Arbeitstage am Stück;

- keine Sparmassnahmen auf dem Rücken dieser Arbeitnehmer und

- es müssen Arbeitszeitmodelle geschaffen werden, welche die Entwicklungen unseres Berufsstandes - höherer Frauenanteil, Kombinierbarkeit von Beruf und Familie, Wunsch nach Teilzeitarbeit - berücksichtigen.

Assistenz- und Oberärztinnen und -ärzte sind nach wie vor mit Begeisterung und viel Engagement im Einsatz. Dies sollte in unser aller Interesse auch zukünftig so bleiben.

Dr. med. Gert Printzen, Mitglied des Zentralvorstandes, Verantwortlicher Ressorts Heilmittel und Angestellte Ärzte

1 Von dem Knesebeck O, Klein J, Frie KG, Blum K, Siegrist J. Psychosoziale Arbeitsbelastungen. Deutsches Ärzteblatt Int. 2010;107(14):248-53. 